\title{
Our solar system as model for exosolar planetary systems
}

\author{
Rudolf Dvorak $^{1}$, Áron Süli ${ }^{2}$ and Florian Freistetter ${ }^{1}$ \\ ${ }^{1}$ Institute for Astronomy, University of Vienna, Türkenschanzstrasse 17, A-1180 Vienna, \\ Austria \\ email: dvorak@astro.univie.ac.at \\ ${ }^{2}$ Department of Astronomy, Eötös University H-1117 Budapest, Pázmány Péter sétány 1/A, \\ Hungary \\ email: a.suli@astro.elte.hu
}

\begin{abstract}
We investigate the dynamical behaviour of a simplified model of our planetary system (Mercury and the planets Uranus and Neptune were excluded) when we change the mass of the Earth via a mass factor $\kappa_{E} \in[1,300]$. This is done to study the motions in this "model planetary system" as an example for extrasolar systems. It is evident that the new systems under consideration can only serve as a model for a limited number of exosystems because they have massive planets sometimes with large orbital eccentricities. We did these numerical experiments using an already well tested numerical integration method (LIE-integration) in the framework of the Newtonian equations of motions. We can show that these planetary systems are very stable up to several hundred earth masses, but for some specific values of $\kappa_{E}$ they show a typical chaotic behaviour already in the semi-major axis. It is know from the inner Solar System that the planets move in a small region of weak chaos, but this behaviour (close to $\kappa_{E}=5$ ) was quite unexpected. We then use a $1^{\text {st }}$ order secular theory to explain the appearance of chaos. The results may serve for a better understanding of the dynamics of some extrasolar planetary systems.
\end{abstract}

Keywords. Solar system: dynamical evolution, extrasolar planetary system

\section{Introduction}

Although we do not have an analytical proof up to now, all numerical and semianalytical results indicate, that our planetary system is dynamically stable for the lifetime of the Solar System (e.g. Ito \& Tanikawa 2002). From their and other results we can see that the large planets exhibit remarkable stability of their orbital parameters on gigayear time scales. On the contrary for the inner Solar System with the 4 terrestrial like planets we have evidence that they are in a zone of weak chaos (Laskar 1990, 1994, 1996). The cause of this chaotic behaviour is now partly understood as a consequence of secular resonances between the motions of the perihelia and the nodes.

With the detection of extrasolar planetary systems (we now know more than 120 extrasolar planets $\dagger$ ) the investigations of the dynamics of our own planetary systems became more important. Although the orbital parameters are quite different in what concerns the eccentricity (some planets move on very eccentric orbits) our own planetary system may serve as a model for the dynamics of stable planetary systems.

In a previous paper (Dvorak \& Süli 2002) the dynamical evolution of a simplified Solar System consisting of the Sun, Venus, Earth, Mars, Jupiter and Saturn was studied; the

$\dagger$ http://www.obspm.fr/encycl/catalog.html 


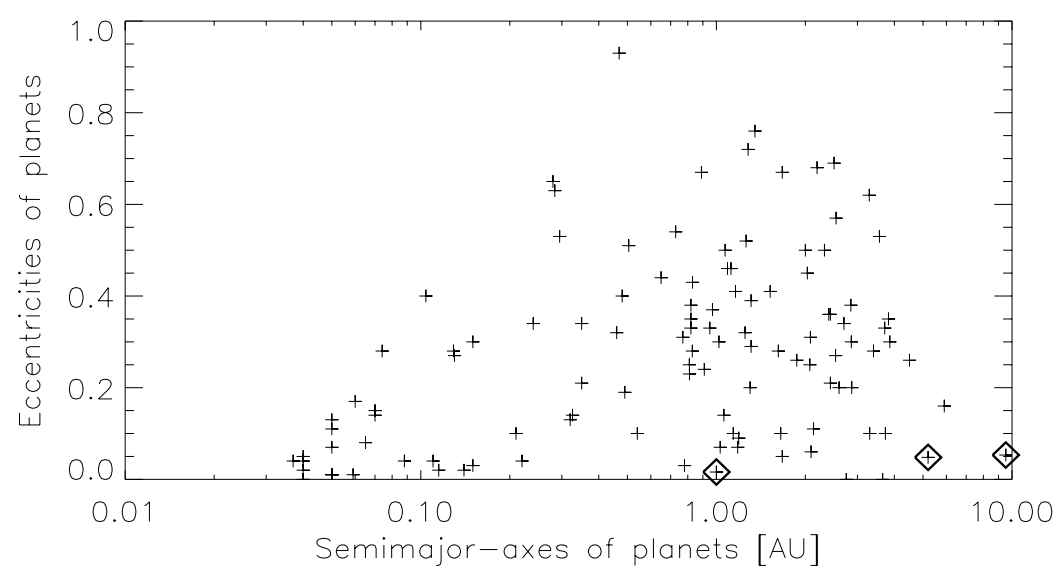

Figure 1. Eccentricity vs. the semi-major axis for extrasolar planets. The $x$-axis is logarithmic. The position of the Earth, Jupiter and Saturn are also indicated as diamonds with a plus sign in the middle.

masses of the inner planets were uniformly magnified via a mass factor $\kappa$. The surprising result was that in this model all the orbits were still stable for time scales of $10^{7}$ years up to $\kappa<245$. In this new investigation we used the same dynamical model, but only the mass of the Earth was multiplied by $\kappa_{E} \in[1,300]$.

In Section 2 we discuss some characteristics of extrasolar planetary systems, Section 3 presents the dynamical model, in Section 4 some results of the dynamics of our planetary system together with the interesting motion of the node of Mars are shown. In Section 5 we show the results of the numerical experiments with a larger mass of the earth together with more detailed results for $\kappa_{E}=5$ and finally we summarise the investigation shortly in Section 6.

\section{Some characteristics of extrasolar planetary systems}

The stability studies got a new impulse because of the recent findings of other planetary systems around single and even double stars. About 120 exoplanetary systems (exosystems) are now known. The so far discovered exoplanets have a mass range $\left(m \cdot \sin \left(i_{p}\right)\right)$ from $0.11 m_{J}$ to $17.5 m_{J}$ where $m_{J}$ is Jupiter's mass and $i_{p}$ is the inclination of the orbital plane with respect to the plane of the sky. More than $90 \%$ of these planets are orbiting 'their sun' well inside Jupiter's orbit ( $a \leqslant 3.3 \mathrm{AU})$. Their semi-major axes accumulate around $1 \mathrm{AU}$ which is a matter of the analysis of observations of the radial velocity curve over periods in the order of years. We can see from Fig. 1 that most of them have significant eccentricities (some $70 \%$ of their orbital eccentricities are larger than $e=0.2$ ). Nevertheless our planetary system may serve as model case for the ones with small eccentricities which are also the ones where we may expect stable terrestrial planets moving in habitable zones (e.g. Ashgari et al. 2004).

\section{The dynamical model and the methods of investigation}

The dynamical model which we studied consisted of the Sun, Venus, Earth, Mars, Jupiter and Saturn. We have chosen this model to minimise the required CPU time for the integration of the - purely Newtonian - equations of motion. Because of the small 
mass of Mercury, it only slightly perturbs the motion of the other planets, but it would require a time step four times smaller than without the innermost planet. Although Uranus and Neptune are massive planets, they orbit the Sun more than twice as far as Saturn, and do not influence the motion of the inner planets significantly. This model was tested already for other numerical computation of the orbits of the near earth asteroids (e.g. Dvorak and Freistetter 2000) and turned out to be quite accurate.

Our method used to solve the equations of motion was the Lie-integration, a method which uses the property of recurrence formulae for the Lie-terms (up to the order 14); one of its advantages is that also high eccentric orbits are integrated accurately due to the automatic step size. A detailed description can be found in Hanslmeier and Dvorak (1984) and Lichtenegger (1984). Many numerical results were derived with this integrator (e.g. Dvorak and Tsiganis 2000, Tsiganis et al. 2000) where the method was also compared to other numerical integrators. The total CPU time used on a high end PC (AMD 2600+ and comparable) was more than 200 days for our study. The interval of data output was set to 100 years which led to a total amount of data of approximately 6 GBs. Complementary we used a secular perturbation theory up to the first order which was implemented in MAPLE (Süli 2003). The comparison of analytical method and numerical results showed quite a good agreement $†$.

\section{Description of the our actual planetary systems}

It is well known that the character of the variation of planetary orbital elements does not change significantly even over the course of long-term simulations over hundreds of million years. The variations of the semi-major axes are very small, for the inner planets it is less than a $0.001 \mathrm{AU}$, for the outer ones it is less than $0.01 \mathrm{AU}$. We know from the work of Laskar that the inner Solar System behaves in a chaotic manner and that there are critical angles which change from circulation to libration and vice-versa on time scales of tens of million years.

We do not have results for such long times in our recent study but we found a very interesting behaviour of $\Omega$, the longitude of the ascending node of Mars. If we compare the time evolution of the orbital element $i$ with $\Omega$, it turns out, that whenever $i_{M a r s}<i_{c r}$, then $\Omega_{\text {Mars }}$ begins to librate around $105^{\circ}$. The center of the temporarily libration of $\Omega_{\text {Mars }}$ coincides with those of Jupiter $\left(90^{\circ}<\Omega_{\text {Jupiter }}<120^{\circ}\right)$ and of Saturn $\left(75^{\circ}<\right.$ $\Omega_{\text {Saturn }}<140^{\circ}$ ). The same behaviour can be observed in the case of Venus and Earth (which we do not show here) although there it is not so obvious in the respective plots.

The above phenomena is very well visible in Figs. 2 and 3, where $\Omega$ and $i$ of Mars are plotted versus the time. It is already reproduced by the first order secular theory. We have used the well known Laplace-Lagrange theory for this system (Süli 2003): a comparison of Figs. 2 and 3 with Fig. 4 shows that the periods of the appearance of libration between numerical and analytical results agree qualitatively but not quantitatively $\left(P_{\text {num }} \sim 2 P_{\text {theory }}\right)$. The reason for the above phenomena is the following: if the reference frame is chosen such that the $x, y$ plane is orthogonal to the total angular momentum vector (i.e. it coincides with the invariant plane) than all the nodes circulate. If the reference frame is chosen sufficiently inclined with respect to the invariant plane, some of the nodes appear to librate. This is the case of Jupiter's and Saturn's node in our ecliptic reference frame, and the reason for the temporarily libration of Mars' node. This phenomena is very well visible in Fig. 4 , where $\Omega$, and $i \cdot 10$ of Mars are plotted versus

$\dagger$ For the initial planetary orbital elements we have chosen for JD 2449200.5 with respect to the mean ecliptic and equinox J2000 from the JPL ephemerides DE200. (Standish 1990). 


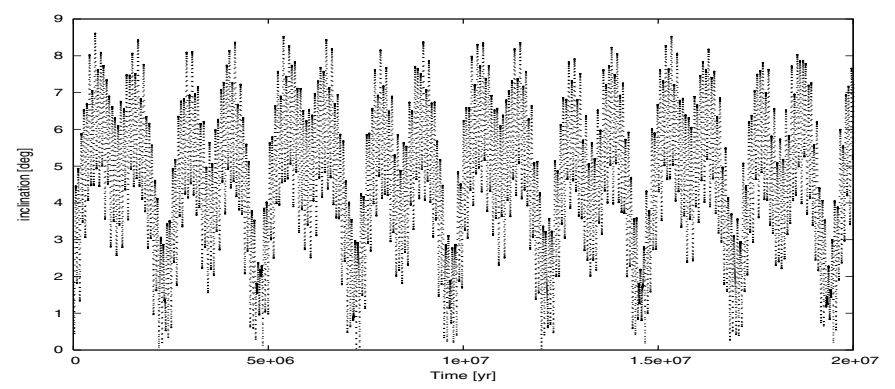

Figure 2. Inclination for Mars for our planetary system versus the time for 20 million years.

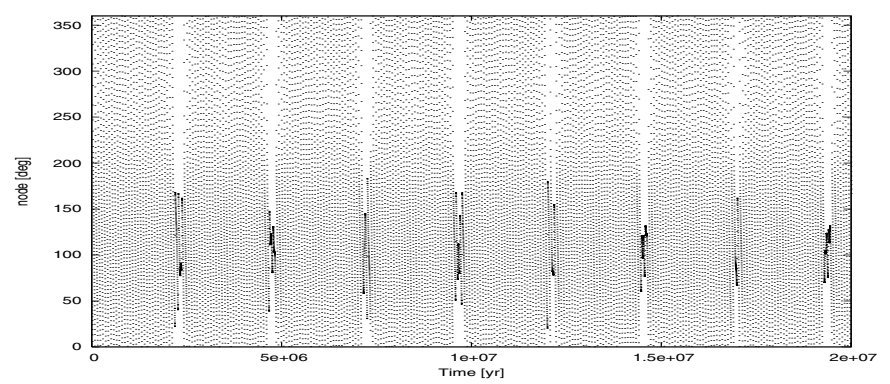

Figure 3. Longitude of the node of Mars for our planetary system for 20 million years; note that the libration modes coincide exactly with the minimum values of the inclinations.

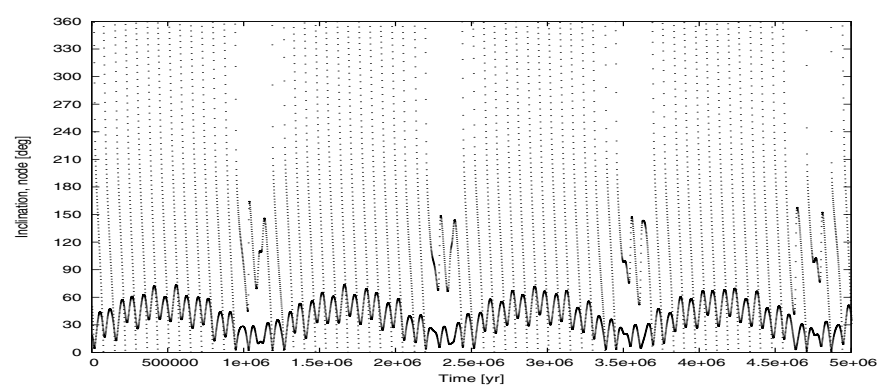

Figure 4. Longitude of the node and inclination (ten times larger) of Mars for 5 Million years by a first order secular theory. Note that the periodic changes from circulation to libration have only half the period of the numerically derived results.

the time. The $i_{c r}$ is related to the inclination of the $x, y$ plane relative to the invariant plane. $\dagger$

We emphasize that the same features are also present when integrating the equations of motion with all 8 massive planets; it characterises the motion of Mars for at least 100 million years into the future and into the past (Dvorak and Gamsjaeger 2003). In the respective plot for Mars (Fig. 5) we see the evolution of the inclination for the first 10 million years together with the evolution of the node. The period is quantitatively the same as in the simplified model. For the time between 90 and 100 million years we found that the change from libration to circulation and vice versa is NOT periodic any more as a consequence of the chaotic nature of the inner planets.

$\dagger$ In the first order theory high order terms are ignored and the change from libration to circulation is sensitive with respect to the periods in the inclination of Mars; this causes the difference in the periods between numerical results and the theory. 

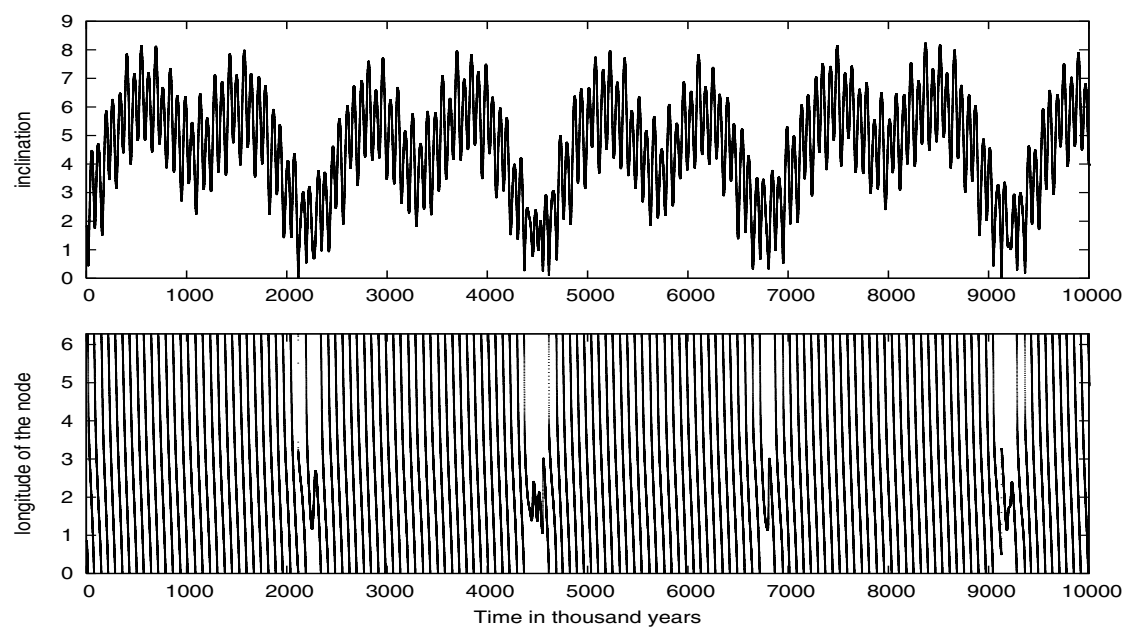

Figure 5. Inclination (upper graph) and longitude of the node (in radians) (lower graph) of Mars for 10 million years after an accurate numerical integration of the complete planetary system. Note that the periodic changes from circulation to libration have the same period as the one in the simplified system.

\section{Numerical experiments with a very massive Earth}

Our aim was to study the stability of the system with respect to the mass of the Earth which may be taken as an adequate model for some of the known exoplanetary system. This kind of a model planetary system is of prior interest for systems where Trojans planets may move on low eccentric orbits in habitable zones; we may even take it then as a reference planetary system! We therefore magnified the mass of the Earth by a mass factor between $1 \leqslant \kappa_{E} \leqslant 300$. Between $1 \leqslant \kappa_{E} \leqslant 15$ the step in $\kappa_{E}$ was one, then up to $\kappa_{E}=100$ the step size was 5 , then up to $\kappa_{E}=300$ the step size was 10 . For $\kappa_{E} \sim 90$ the mass of Earth is comparable to the gas giant Saturn, for $\kappa_{E} \sim 300$ to Jupiter; then Venus and Mars can be considered as being protoplanets with relatively small masses. These resulting systems may be considered as models for 47 Ursae Majoris with its three massive planets, when we multiply the semi-major axes of this extrasolar system by 2.5 . In Fig. 6 we show how the masses of the planets compare when we introduce the mass factor $\kappa_{E}$ for the Earth. In Fig. 7 also the mass distribution of the known exoplanetary systems is shown as a function of the mass parameter $\left(M_{J u p} \sin i\right)$.

We expected that Venus but especially Mars will suffer more and more from the perturbations due to a more massive Earth. To quantify these effects we checked the orbital element eccentricity during the whole integration time of $2 \cdot 10^{7}$ years for every model and every planet. We determined the maximum value of the eccentricity $(=\mathrm{ME})$ which is an important parameter for the dynamical evolution and stability of the system. It is a reliable indicator of chaos and the maximum eccentricity method (MEM) was already used successfully in many numerical investigations concerning the dynamics of planetary systems (e.g. Dvorak et al. 2003). The respective plot of ME for the whole range is shown in Fig. 7 (left panel), where no effect on Jupiter and Saturn is visible. We note that the ME of Earth for $\kappa_{E}=1$ is $60 \%$ greater than for $\kappa_{E}=2$, and it decreases further as the mass factor increases. The ME of Venus shows a similar behaviour as a consequence of the strong coupling between Venus and Earth; we observe two larger values of the ME of Venus, which are still rather small and do not influence the stability of the system. 


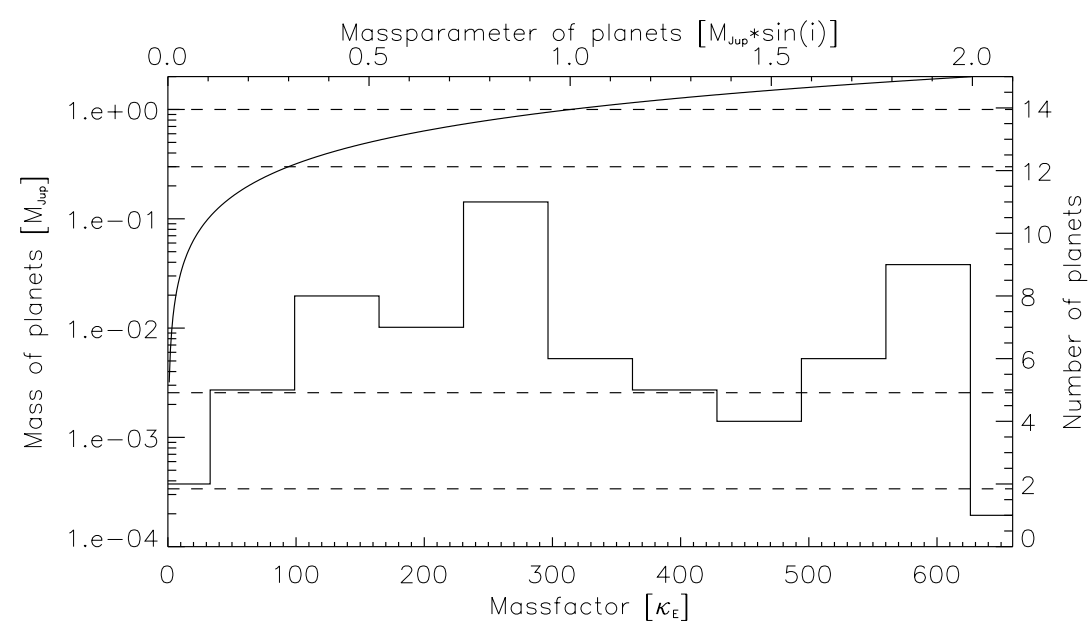

Figure 6. The mass distribution of the known exoplanetary systems are plotted together with the mass of the Earth as a function of the mass factor $\left(\kappa_{E}\right)$ (solid line). The masses of the other planets were unchanged, and are represented by straight dashed lines (units are Jupiter masses). On the lower $\mathrm{x}$-axis the mass factor is scaled; the upper $\mathrm{x}$-axis shows the scaling of the mass parameter of the exoplanetary system. The left y-axis is the mass of the planets in a logarithmic scale; the right $\mathrm{y}$-axis is the number of planets. The bin size is $0.2 \mathrm{~m}_{\text {Jupiter }}$.
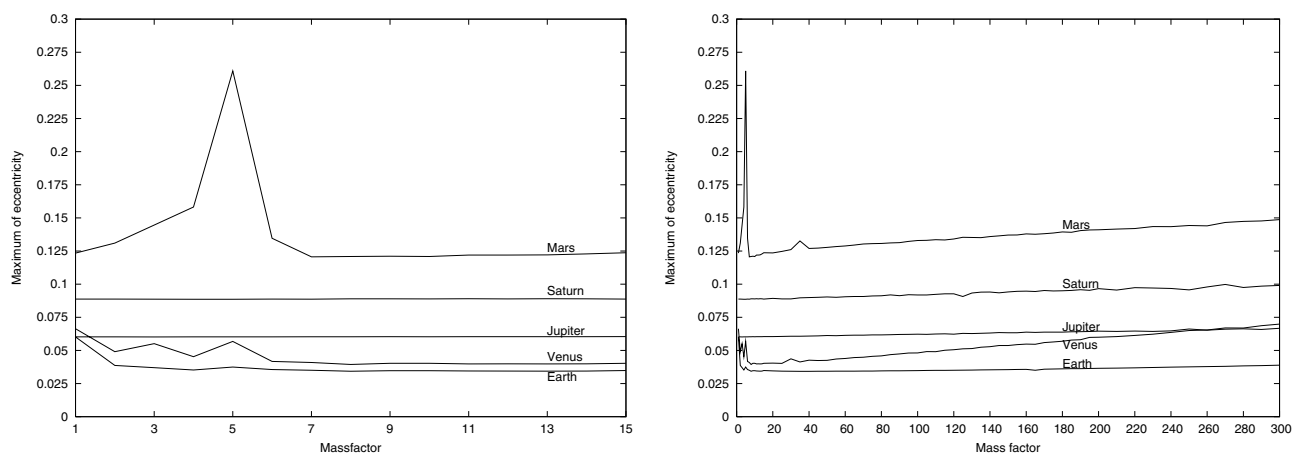

Figure 7. Results of the MEM: the maximum eccentricity as a function of $\kappa_{E}$. On the left graph $1 \leqslant \kappa_{E} \leqslant 15 ;$ on the right graph $1 \leqslant \kappa_{E} \leqslant 300$

Then, for larger values of $\kappa_{E}>15$ one can see a gradual but small increase of Venus' ME (see Fig. 7, right panel).

Mars is playing a special role (Fig. 7, left panel): the ME of Mars steadily increases with $\kappa_{E}$, and at $\kappa_{E}=5$, it suddenly reaches a very high value $\left(e_{\text {Mars }}=0.26\right.$, perihelion distance $q=1.13 \mathrm{AU}$ ). After this peak the ME drops down to its starting value, and stays around 0.123 . Beyond $\kappa_{E}=15$ Mars' ME linearly increases with the mass factor.

Checking the elements of Mars for $\kappa_{E}=5$ we can see that the semi-major axes shows an interesting non periodically modulation, which is also visible from the plot of the eccentricity (Fig. 8). The eccentricity increases rapidly to $e=0.26$ and shows a periodic large variation $0.07<e<0.26$, but after several million years stays rather large $0.19<$ $e<0.26$. To explain this special behaviour we used again the first order secular theory, although we know that for larger eccentricities it will fail to provide precise results. Nevertheless we found the reason for this irregular behaviour: Table 1 shows the analysis of the fundamental frequencies involved. We observe the closeness of the two frequencies 

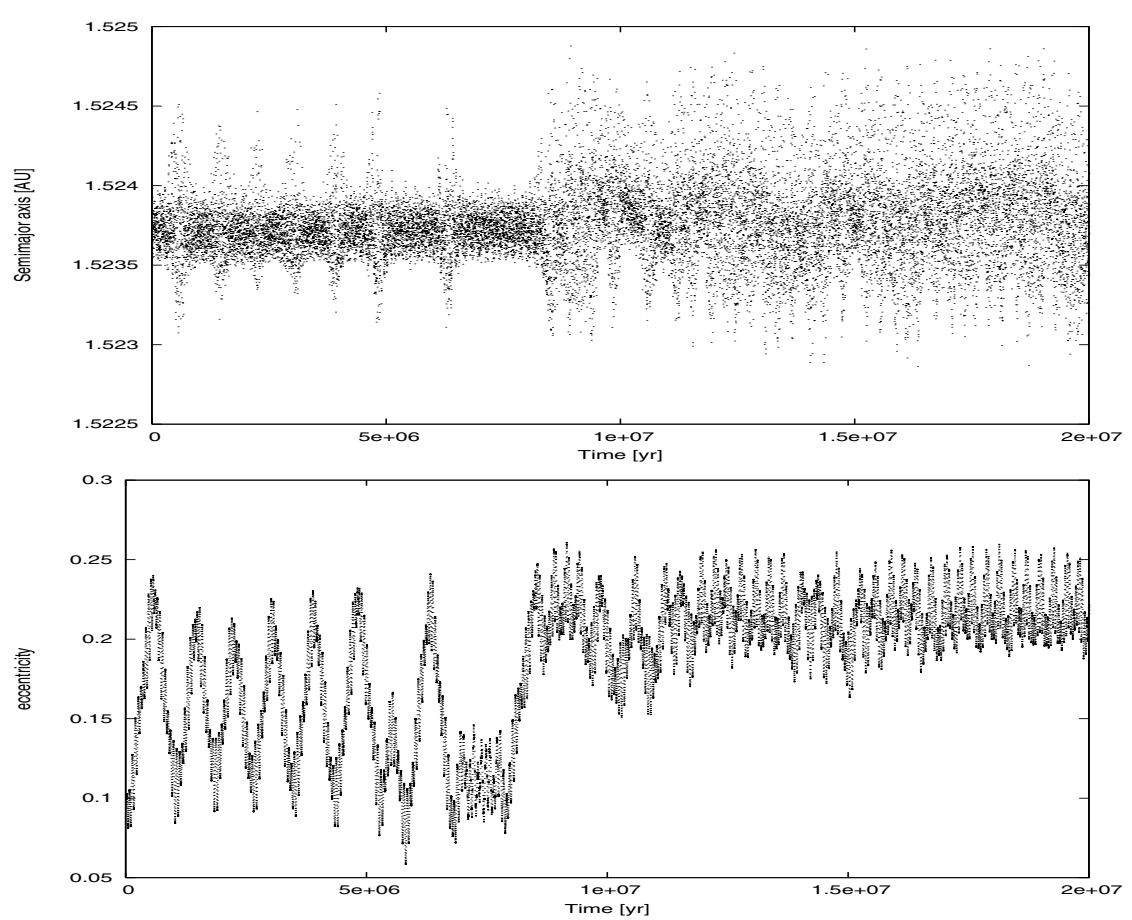

Figure 8. Semi-major axis (top graph) and eccentricity (bottom graph) of the system with $\kappa=5$ for $2 \cdot 10^{7}$ years

$f_{2}$ and $f_{3}$ which shows that they are almost in a 1:1 resonance! A thorough investigation of the planetary systems around the value of $\kappa_{E}=5$ using numerical and analytical tools is in progress. We hope to give then an even more detailed explanation of this interesting and - up to now - not known dynamical behaviour of our slightly modified planetary system.

Table 1. The secular frequencies of the model for $\kappa_{E}=5$ calculated using the Lagrange-Laplace secular theory. The units are in $\operatorname{arcsec} / y r$, year and in degrees.

\begin{tabular}{rrrr|rrr|}
\hline$j$ & $g_{j}[" / \mathrm{yr}]$ & $P_{j}[\mathrm{yr}]$ & $\beta_{j}[\mathrm{deg}]$ & $f_{j}[" / \mathrm{yr}]$ & $P_{j}[\mathrm{yr}]$ & $\gamma_{j}[\mathrm{deg}]$ \\
\hline 1 & 3.540609 & 366038.71 & 290.97 & -47.160423 & 27480.67 & 76.93 \\
2 & 8.679525 & 149316.93 & 109.16 & -25.838731 & 50157.26 & 306.49 \\
3 & 22.284358 & 58157.39 & 23.72 & -25.682030 & 50463.30 & 129.59 \\
4 & 25.686232 & 50455.05 & 116.03 & -7.155340 & 181123.47 & 297.68 \\
5 & 45.645800 & 28392.54 & 304.59 & 0 & - & - \\
\hline
\end{tabular}

\section{Conclusions}

We studied the dynamics of a simplified dynamical model of the Solar System to have a kind of general model of exoplanetary systems with planets on not too eccentric orbits. We found an interesting periodic change from circulation to libration of the Mars' longitude of the node (and vice versa) what we have explained as a consequence of the inclination of the ecliptic reference frame with respect to the invariant plane. 
We then studied in the same model the orbits of the planets when we increase the mass of the Earth by a mass factor between $1 \leqslant \kappa_{E} \leqslant 300$. These model systems were still stable even when the Earth had Jupiter's mass; older, not published results, showed that from approximately $m_{\text {Earth }} \sim 3 M_{\text {Jupiter }} \sim \kappa_{E}=1000$ on close approaches of Mars and Venus to the Earth made the system dynamically unstable.

For $\kappa_{E}=5$ signs of chaotic motion appeared in the semi-major axis of Mars, whose orbital eccentricity reached values up to $e=0.256$ during the integration time of $2 \cdot 10^{7}$ years. Using the results of the Lagrange-Laplace secular theory to the first order for $\kappa_{E}=5$, we found a secular resonance acting between the motions of the nodes of the Earth and Mars. According to these results, the stability of the Solar System depends strongly on the masses of the planets, and small changes in these parameters result in a different dynamical evolution of the planetary system. Therefore we think that this planetary system may be used as dynamical reference model for a better understanding of the orbits in extrasolar systems.

\section{Acknowledgements}

Most of the numerical integrations were accomplished on the NIIDP (National Information Infrastructure Development Program) supercomputer in Hungary. F. Freistetter acknowledges the support by the Austrian FWF project P16024. Á. Süli is grateful to the Hungarian NRF (grant no. OTKA T043739).

\section{References}

Asghari, N., Broeg, C., Carone, L., Casas-Miranda, R., Palacio, J. C. Castro Csillik, I., Dvorak, R., Freistetter F. and 33 more authors 2004, Astron. Astrophys., in press.

Dvorak, R. and Gamsjäger, C. 2003, in: F. Freistetter, R. Dvorak, B. Érdi (eds.), Proceedings of the $3^{\text {rd }}$ Austrian Hungarian workshop on trojans and related topics, p. 49

Dvorak, R., Pilat-Lohinger, E., Funk, B. and Freistetter, F. 2003, Astron. Astrophys., 398, L1

Dvorak, R. and Süli, Á. 2002, Cel. Mech. Dyn. Astron., 83, 77

Dvorak, R. and Tsiganis, K. 2000, Cel. Mech. Dyn. Astron., 78, 125

Dvorak, R. and Freistetter, F. 2000, Planet. Space Sci., 49, 803

Hanslmeier and A., Dvorak, R. 1984, Astron. Astrophys., 132, 203

Ito, T. and Tanikawa, K. 2002, Mon. Not. R. Astron. Soc., 336, 483

Laskar, J. 1990, Icarus, 88, 266

Laskar, J. 1994, Astron. Astrophys., 287, L9

Laskar, J. 1996, Cel. Mech. Dyn. Astron., 64, 115

Lichtenegger, H. 1984, Celest. Mech., 34, 357

Süli, Á. 2003, in: F. Freistetter, R. Dvorak, B. Èrdi (eds.), Proceedings of the $3^{\text {rd }}$ Austrian Hungarian workshop on trojans and related topics, p. 85

Tsiganis, K., Dvorak, R. and E. Pilat-Lohinger. 2000, Astron. Astrophys., 354, 1091

Standish, E.M. 1990, Astron. Astrophys., 233, 252 